TOPOLOGICAL ALGEBRAS, THEIR APPLICATIONS,

AND RELATED TOPICS

BANACH CENTER PUBLICATIONS, VOLUME 67

INSTITUTE OF MATHEMATICS

POLISH ACADEMY OF SCIENCES

WARSZAWA 2005

\title{
BOURGAIN ALGEBRAS OF G-DISC ALGEBRAS
}

\author{
T. TONEV and K. YALE \\ The University of Montana, Missoula, MT 59803, U.S.A. \\ E-mails: tonevtv@mso.umt.edu,ikyale@msn.com
}

1. Introduction. The norm topology of a commutative Banach algebra $A$ is too rough to reflect some of the delicate properties of $A$. Weaker topologies are consequently of importance and they can be used to construct algebras associated to $A$ and which contain important information about $A$. Bourgain algebras were introduced by J. Cima and R. Timoney [2] in their study of Dunford-Pettis property $(D P P)$ for a certain class of function algebras. In effect, the algebra $A$ has the $D P P$ whenever its Bourgain algebra is as large as possible. In this paper we determine the Bourgain algebras related with some $G$-disc algebras.

Given a commutative Banach algebra $A$, let $c_{o}^{\tau}(A)$ denote the family of all sequences of elements in $A$ which tend to 0 with respect to a given topology $\tau$ on $A$. For the weak topology $w, c_{o}^{w}(A)$ is the set of all weakly null sequences of elements $\varphi_{n}$ in $A$, i.e. sequences $\left\{\varphi_{n}\right\}$ such that $L\left(\varphi_{n}\right) \rightarrow 0$ as $n \rightarrow \infty$ for any bounded linear functional on $A$.

Let $A \subset B$ be two commutative Banach algebras and let the norm $\|\cdot\|_{A}$ be the restriction of the norm $\|\cdot\|_{B}$ to $A$. The Bourgain algebra $A_{b}^{B}$ of $A$ with respect to $B$ is the set of all $f$ in $B$ such that for every weakly null sequence $\left\{\varphi_{n}\right\}_{n}$ in $A$ there exist a sequence $\left\{g_{n}\right\}_{n}$ in $A$ such that $\left\|f \varphi_{n}-g_{n}\right\|_{B} \rightarrow 0$ as $n \rightarrow \infty$ [2].

Let $\pi_{A}: B \rightarrow B / A$ be the natural projection of $B$ onto $B / A$. For every fixed $f \in B$ let $P_{f}: A \rightarrow f A \subset B$ be the multiplication by $f \in B$ on $A$. Denote $S_{f}=\pi_{A} \circ P_{f}: A \rightarrow$ $(f A+A) / A \subset B / A$ the Hankel type operator $S_{f}: g \mapsto \pi_{A}(f g)$. Note that $\pi_{A}$ and $S_{f}$ both are bounded linear maps onto $B / A$ and onto $(f A+A) / A \subset B / A$ correspondingly.

Observe that $f \in A_{b}^{B}$ if $S_{f}$ maps every weakly null sequence of $A$ onto a null sequence with respect to the quotient norm topology of $\pi_{A}(f A) \subset B / A$. Thus $f \in A_{b}^{B}$ if the operator $S_{f}$ is completely continuous, i.e. $S_{f}\left(\varphi_{n}\right)=\pi_{A}\left(f \varphi_{n}\right) \rightarrow 0, n \rightarrow \infty$ for every

2000 Mathematics Subject Classification: Primary 46J10; Secondary 30G30, 43A75, 30D55.

Key words and phrases: Bourgain algebra, finite-dimensional Banach space, completely continuous operator, generalized analytic function, the big disc algebra.

Research partially supported by grants from the NSF and the University of Montana.

The paper is in final form and no version of it will be published elsewhere. 
weakly null sequence $\left\{\varphi_{n}\right\}_{n}$ in $A$. Equivalently, $f \in A_{b}^{B}$ if and only if $S_{f}\left(c_{o}^{w}(A)\right) \subset$ $c_{O}^{\|\cdot\|}(B / A)$.

Let $A_{w c}^{B}$ be the set of all $f$ in $B$ such that $S_{f}$ is weakly compact (rather than completely continuous). In a uniform algebra setting, B. Cole and T. W. Gamelin [3] introduced the notion of tightness related with the space $A_{w c}^{B}$. A precise connection between $A_{b}^{B}$ and $A_{w c}^{B}$ is not known (cf. [9]).

2. Hankel type operators and Bourgain algebras of $G$-disc algebras. Let $A \subset B$ be two uniform algebras on a compact Hausdorff set $X$ and let $A_{b}^{B}$ be the Bourgain algebra of $A$ with respect to $B$.

Proposition 1. If the range $S_{f}(A)=\pi_{A}(f A)$ of the Hankel type operator $S_{f}$ for an $f \in B$ is finite dimensional then $f \in A_{b}^{B}$.

Proof. If $\left\{\varphi_{n}\right\}_{n}$ is a weakly null sequence in $A$ then $\left\{f \varphi_{n}\right\}_{n}$ is weakly null in $B$, and therefore $\left\{\pi_{A}\left(\varphi_{n}\right)\right\}_{n}$ is a weakly null sequence in $\pi_{A}(f A) \subset B / A$. Hence $\left\{\pi_{A}\left(\varphi_{n}\right)\right\}_{n} \in$ $c_{0}^{\|\cdot\|}\left(\pi_{A}(f A)\right) \subset c_{0}^{\|\cdot\|}(B / A)$, since $\pi_{A}(f A)$ is finite dimensional. Consequently $f \in A_{b}^{B}$.

The range of the completely continuous operator $S_{f}$ need not be finite-dimensional. The following example is due to S. Saccone.

Example 1 . Let $A=A(\mathbb{T})$ be the disc algebra on the unit circle $\mathbb{T}$ and let $B=C(\mathbb{T})$. Consider the function

$$
f(z)=\sum_{k=1}^{\infty} \frac{1}{k^{2} z^{k}} .
$$

Since $f \in C(\mathbb{T})$, it certainly belongs to $A_{b}^{B}$. We claim that the range of the Hankel type operator of $f$ is infinite dimensional. Indeed, let $c_{n}=\left\|z^{n} f+A\right\|_{B / A}$, and let $g_{n}(z)=$ $\left(1 / c_{n}\right) z^{n}$. Clearly, $g_{n} \in A$, and $\left\|g_{n} f+A\right\|_{B / A}=1$. To see that $\pi_{A}(f A)$ is not finite dimensional it is enough to show that $g_{n} f+A$ converges weakly to zero in $B / A$.

The value of the $(-m)$-th Fourier coefficient of the function $g_{n} f$ is

$$
\int_{\mathbb{T}} g_{n}(z) f(z) z^{m} d z=\frac{1}{c_{n}(n+m)^{2}}, \quad m, n \geq 1 .
$$

Hence,

$$
c_{n} \geq\left\|z^{n} f+H^{2}\right\|_{L^{2} / H^{2}}=\sqrt{\sum_{k=1}^{\infty} \frac{1}{(n+k)^{4}}},
$$

thus

$$
\frac{1}{n^{2} c_{n}} \leq \frac{1}{\sqrt{n^{4} \sum_{k=1}^{\infty} \frac{1}{(n+k)^{4}}}}
$$

Furthermore,

$$
\sum_{k=1}^{\infty} \frac{1}{(n+k)^{4}} \geq \int_{n+1}^{\infty} \frac{1}{x^{4}} d x=\frac{1}{3(n+1)^{3}}
$$


So

$$
n^{4} \sum_{k=1}^{\infty} \frac{1}{(n+k)^{4}} \rightarrow \infty
$$

as $n \rightarrow \infty$. Hence, $\lim _{n \rightarrow \infty} 1 /\left(n^{2} c_{n}\right)=0$, and therefore we have

$$
\lim _{n \rightarrow \infty} \int_{\mathbb{T}} g_{n}(z) f(z) z^{m} d z=\lim _{n \rightarrow \infty} \frac{1}{c_{n}(n+m)^{2}}=0
$$

for all $m \in \mathbb{N}$. It now follows that if $p$ is any polynomial with $p(0)=0$, then

$$
\lim _{n \rightarrow \infty} \int_{\mathbb{T}} g_{n}(z) f(z) p(z) d z=0 .
$$

Recall that if $X$ is a Banach space and $\left\{x_{n}\right\}_{n}$ is a bounded sequence in $X$ tending to zero on a norm-dense set of the dual space $X^{*}$, then $\left\{x_{n}\right\}_{n}$ is weakly null. Since the space $H_{0}^{1}$ is isometrically isomorphic to $(C(\mathbb{T}) / A(\mathbb{T}))^{*}$, and the polynomials $p$ with $p(0)=0$ are dense in $H_{0}^{1}, g_{n} f+A$ converges weakly to zero in $B / A$, as claimed.

Let $G$ be a compact abelian group with identity $e$ and let $S \subset \widehat{G} \subset C(G)$ be a subsemigroup of the dual group $\widehat{G}$ containing the unit $1_{\widehat{G}}=1_{S}$. For a fixed character $\chi \in \widehat{G}$ denote by $\mathcal{P}_{\chi}$ the set $\chi S \backslash S$. The uniform algebra on $G$ generated linearly by the semigroup $S$ will be denoted by $A_{S}$. Functions in $A_{S}$ are called $S$-functions on $G$ ([4, Ch. VII], [5], [6, Ch. II]).

Proposition 2. Any character $\chi \in \widehat{G}$ for which $\mathcal{P}_{\chi}$ is finite belongs to $\left(A_{S}\right)_{b}^{C(G)}$.

Proof. Note that the characters on $G$ are linearly independent in $C(G)$. Since the algebra $A_{S}$ is generated linearly by $S \subset C(G)$, the sets $\mathcal{P}_{\chi}$ and $\pi_{A_{S}}\left(\mathcal{P}_{\chi}\right)$ have the same cardinality. Therefore,

$$
\operatorname{dim}\left(S_{\chi}\left(A_{S}\right)\right)=\operatorname{dim}\left(\pi_{A_{S}}\left(\chi A_{S}\right)\right)=\operatorname{card}\left(\pi_{A_{S}}\left(\mathcal{P}_{\chi}\right)\right)=\operatorname{card}\left(\mathcal{P}_{\chi}\right)<\infty .
$$

By Proposition 1 the Hankel type operator $S_{\chi}$ is completely continuous. Hence $\chi$ belongs to $\left(A_{S}\right)_{b}^{C(G)}$ as claimed.

Note that for any $\chi \in S$ the set $\mathcal{P}_{\bar{\chi}}$ has the same cardinality as $\chi \mathcal{P}_{\bar{\chi}}=S \backslash \chi S=\{\gamma \in$ $S: \gamma \notin \chi S\}$, which is the set of all predecessors of $\chi$ in $S$, i.e. of all elements $\gamma$ in $S$ which precede $\chi$ with respect to the ordering on $\widehat{G}$ determined by $S$. If, in addition, $S-S=\widehat{G}$ and every $\chi \in S$ has finitely many predecessors in $S$ then every character $\chi \in \widehat{G}$ has finitely many predecessors in $S$. As it follows from Proposition 2, then $\left(A_{S}^{C(G)}\right)_{b}=C(G)$, and therefore the corresponding algebra $A_{S}$ possesses the Dunford-Pettis property.

Corollary 1. If $\chi \in S$ be such that $S \backslash\left\{1_{S}\right\} \subset \chi S$, then $\bar{\chi} \in\left(A_{S}\right)_{b}^{C(G)}$.

Proof. Since $\chi \mathcal{P}_{\bar{\chi}}=S \backslash \chi S=\left(\left\{1_{S}\right\} \cup\left(S \backslash\left\{1_{S}\right\}\right)\right) \backslash \chi S \subset\left(\left\{1_{S}\right\} \cup \chi S\right) \backslash \chi S=\left\{1_{S}\right\}$, we obtain that $\mathcal{P}_{\bar{\chi}}=\{\bar{\chi}\}$. Hence $\bar{\chi} \in\left(A_{S}\right)_{b}^{C(G)}$ by Proposition 2 .

Corollary 2. If $A_{S}$ is a maximal algebra and the set $\mathcal{P}_{\chi}$ is finite for some character $\chi \in \widehat{G} \backslash S$, then $\left(A_{S}\right)_{b}^{C(G)}=C(G)$.

Proof. Indeed, $\chi \in\left(A_{S}\right)_{b}^{C(G)}$ by Proposition 2. Since $\chi \notin S$, then $\chi \notin A_{S}$ and consequently $\left(A_{S}\right)_{b}^{C(G)}=C(G)$ by the maximality of $A$. 
EXAMPLE 2. If $H$ is a finite group, $G=(H \oplus \mathbb{Z})^{\wedge}$ and $S \cong H \oplus \mathbb{Z}_{+}$, then $\left(A_{S}\right)_{b}^{C(G)}=$ $C(G)$.

Indeed, for every character $\chi_{(h, n)} \in \widehat{G}$, where $h \in H$ and $n \in \mathbb{Z}$, we have

$$
\begin{gathered}
\operatorname{card}\left(\mathcal{P}_{\chi_{(h, n)}}\right)=\operatorname{card}\left((h, n)\left(H \oplus \mathbb{Z}_{+}\right) \backslash H \oplus \mathbb{Z}_{+}\right)= \\
\operatorname{card}\left(\left(h H \oplus\left(n+\mathbb{Z}_{+}\right)\right) \backslash H \oplus \mathbb{Z}_{+}\right)=\operatorname{card}\left(\left(H \oplus\left(n+\mathbb{Z}_{+}\right)\right) \backslash H \oplus \mathbb{Z}_{+}\right)= \\
\operatorname{card}\left(H \oplus\left(\left(n+\mathbb{Z}_{+}\right) \backslash \mathbb{Z}_{+}\right)\right)=\operatorname{card} H+n<\infty .
\end{gathered}
$$

By Proposition 2 we see that $\chi_{(h, n)} \in\left(A_{S}\right)_{b}^{C(G)}$ for every $h \in H$ and $n \in \mathbb{Z}$. Consequently $\widehat{G}=H \oplus \mathbb{Z} \subset\left(A_{S}\right)_{b}^{C(G)}$, wherefrom $\left(A_{S}\right)_{b}^{C(G)}=C(G)$.

In the sequel we will assume that $S \cup(-S)=\widehat{G}=\left\{\chi^{a}\right\}_{a \in \Gamma}$, for some subgroup $\Gamma \subset \mathbb{R}$ that is dense in $\mathbb{R}$, and that $S \cong \Gamma_{+}=\Gamma \cap[0, \infty)$. In this case $A_{S}$ is called the $G$-disc algebra (or, the big disc algebra), and the elements of $A_{S}$ are called also generalized analytic functions on $G$. The following theorem identifies the algebra $\left(A_{S}\right)_{b}^{C(G)}$ for some $G$-disc algebras.

THEOREM 1. If $G$ is a compact abelian group whose dual group $\widehat{G} \cong \Gamma$ is dense in $\mathbb{R}$ and is divisible by an integer $n \in \Gamma$, then the Bourgain algebra $\left(A_{\Gamma_{+}}\right)_{b}^{C(G)}$ of the $G$-disc algebra $A_{\Gamma_{+}}$coincides with $A_{\Gamma_{+}}$.

Without loss of generality we can assume that $1 \in \Gamma_{+}$, thus $1 / n \in \Gamma_{+}$, i.e. $\chi^{\frac{1}{n}} \in \widehat{G}_{+}$. Clearly, $\Gamma_{+}$is a subset of $\left(A_{\Gamma_{+}}\right)_{b}^{C(G)}$. First we will prove two preliminary lemmas.

LEMma 1. The sequence of real valued functions $\varphi_{n}(x)=\left|\frac{1+e^{i \frac{x}{n}}}{2}\right|^{2 n}$ converges pointwise to 1 as $n \rightarrow \infty$ for every $x \in \mathbb{R}$.

Proof. Fix an $x \in \mathbb{R}$. Since $e^{i \frac{x}{n}} \neq-1$ for $n$ big enough, we have

$$
\varphi_{n}(x)=\left(\left|\frac{1+e^{i \frac{x}{n}}}{2}\right|^{2}\right)^{n}=\left(\frac{2+2 \cos \frac{x}{n}}{4}\right)^{n}=\cos ^{2 n} \frac{x}{2 n} \rightarrow 1
$$

as $n \rightarrow \infty$.

Note that the convergence in Lemma 1 is not uniform since, say, $\varphi_{n}(x)=0$ if $x=\pi n$ for any integer $n$.

LEMma 2. Under the setting of Theorem 1 the functions $\psi_{n}(g)=\left|\frac{1+\chi^{\frac{1}{n}}(g)}{2}\right|^{2 n}$ converge pointwise to 1 as $n \rightarrow \infty$ for every $g \in G$.

Proof. Let $j_{e}: \mathbb{R} \rightarrow G$ be the standard embedding of the real line onto a dense subgroup of $G$ such that $j_{e}(0)=e\left(\operatorname{cf}\right.$. [4, Ch. VII], [6, Ch. II]). Then $\chi^{\frac{1}{n}}\left(j_{e}(x)\right)=e^{i \frac{x}{n}}$ and $\psi_{n}\left(j_{e}(x)\right)=\varphi_{n}(x)$ for every real $x$. Hence $\varphi_{n}(x) \rightarrow 1$ as $n \rightarrow \infty$ by Lemma 1 .

Consider the following neighborhood $U$ of $e: U=\left(\chi^{1}\right)^{-1}\left\{e^{i t},-\pi / 4<t<\pi / 4\right\} \subset$ $G$. Note that if $\sqrt[n]{[\cdot]}$ is the principal value of the $n$-th root considered on the set $\left\{e^{i t},-\pi / 4<t<\pi / 4\right\}$, then $\chi^{\frac{1}{n}}(h)=\sqrt[n]{\chi^{1}(h)}$ on $U$. For a given $g \in G$ there is a $h_{g} \in U$ such that $g=j_{h_{g}}(x)$ for some $x \in \mathbb{R}$, where $j_{h}=h j_{e}$ is the standard dense 
embedding of $\mathbb{R}$ into $G$ with $j_{h}(0)=h$. Hence $\chi^{\frac{1}{n}}\left(h_{g}\right)=e^{i \frac{s}{n}}$ if $\chi^{1}\left(h_{g}\right)=e^{i s}$ for some $s,-\pi / 4<s<\pi / 4$, and therefore,

$$
\begin{aligned}
\psi_{n}(g) & =\psi_{n}\left(j_{h_{g}}(x)\right)=\left|\frac{1+\chi^{\frac{1}{n}}\left(j_{h_{g}}(x)\right)}{2}\right|^{2 n} \\
& =\left|\frac{1+\chi^{\frac{1}{n}}\left(h_{g}\right) \chi^{\frac{1}{n}}\left(j_{e}(x)\right)}{2}\right|^{2 n}=\left|\frac{1+e^{i \frac{s+x}{n}}}{2}\right|^{2 n} .
\end{aligned}
$$

Consequently, by Lemma $1, \psi_{n}(g)=\varphi_{n}(s+x) \rightarrow 1$ as $n \rightarrow \infty$.

The remark after Lemma 1 indicates that the convergence in Lemma 2 might not be uniform.

Proof of Theorem 1. Suppose that $\bar{\chi}^{3} \in\left(A_{\Gamma_{+}}\right)_{b}^{C(G)}$, and consider the sequence $\xi_{n}(g)=$ $\psi_{n}(g)-1$, where $\psi_{n}$ is the function in Lemma 2. The sequence $\left\{\chi^{1} \xi_{n}\right\}_{n}$ converges pointwise to 0 on the compact group $G$, and therefore it is weakly null in $A_{\Gamma_{+}}$. Since $\bar{\chi}^{3} \in\left(A_{\Gamma_{+}}\right)_{b}^{C(G)}$, there are functions $h_{n} \in A_{\Gamma_{+}}$such that $\left\|\bar{\chi}^{3} \chi^{1} \xi_{n}-h_{n}\right\|<1 / n$ for every $n$, where $\|\cdot\|$ is the sup norm on $G$. By integrating over $\operatorname{Ker}\left(\chi^{\frac{1}{n}}\right)$, if necessary, we can assume that $h_{n}=q_{n}\left(\chi^{\frac{1}{n}}\right)$ for some polynomial $q_{n}$. Since

$$
\left(\chi^{1} \psi_{n}\right)(g)=\left(\chi^{\frac{1}{n}}(g)\right)^{n}\left(\frac{1+\chi^{\frac{1}{n}}(g)}{2}\right)^{n}\left(\frac{1+\bar{\chi}^{\frac{1}{n}}(g)}{2}\right)^{n}=p_{n}\left(\chi^{\frac{1}{n}}(g)\right),
$$

where $p_{n}$ is the polynomial $p_{n}(z)=\left(\frac{1+z}{2}\right)^{2 n}$, we have that $\chi^{1} \psi_{n} \in A_{\Gamma_{+}}$, and therefore, $\xi_{n} \in A_{\Gamma_{+}}$too. For $j=2 n$ the $j$-th Cesàro mean

$$
\sigma_{j}^{p_{n}}=\frac{S_{0}+S_{1}+\cdots+S_{j}}{j+1}
$$

of $p_{n}$, where $S_{k}$ is the $k$-th partial sum of $p_{n}$, becomes

$$
\sigma_{2 n}^{p_{n}}(z)=\frac{1}{4^{n}(2 n+1)} \sum_{k=0}^{2 n}(2 n-k+1)\left(\begin{array}{c}
2 n \\
k
\end{array}\right) z^{k} .
$$

Hence

$$
\begin{gathered}
4^{n}(2 n+1) \sigma_{2 n}^{p_{n}}(z)=\sum_{k=0}^{2 n}\left(\begin{array}{c}
2 n \\
k
\end{array}\right) z^{k}+\sum_{k=0}^{2 n-1}(2 n-k)\left(\begin{array}{c}
2 n \\
k
\end{array}\right) z^{k}= \\
(1+z)^{2 n}+2 n(1+z)^{2 n-1}=(2 n+1+z)(1+z)^{2 n-1}
\end{gathered}
$$

Now

$$
\begin{aligned}
\left\|\bar{\chi}^{3} \chi^{1} \xi_{n}-h_{n}\right\| & =\max _{g \in G}\left|\left(\bar{\chi}^{3} \chi^{1} \xi_{n}\right)(g)-h_{n}(g)\right| \\
& =\max _{g \in G}\left|\left(\chi^{1} \xi_{n}\right)(g)-\left(\chi^{3} h_{n}\right)(g)\right|=\max _{g \in G}\left|\left(\chi^{1} \psi_{n}\right)(g)-\chi^{1}(g)-\chi^{3}(g) h_{n}(g)\right| \\
& =\max _{g \in G}\left|p_{n}\left(\chi^{\frac{1}{n}}(g)\right)-\chi^{1}(g)-\left(\chi^{\frac{1}{n}}(g)\right)^{3 n} q_{n}\left(\chi^{\frac{1}{n}}(g)\right)\right| \\
& =\max _{z \in \mathbb{T}}\left|p_{n}(z)-z^{n}-z^{3 n} q_{n}(z)\right| .
\end{aligned}
$$

Note that $\sigma_{2 n}^{p_{n}(z)-z^{n}}(z)=\sigma_{2 n}^{p_{n}(z)-z^{n}-z^{3 n} q_{n}(z)}(z)$ because the Cesàro mean $\sigma_{2 n}$ depends only on the first $2 n$ terms of the Taylor series. Since $\max _{z \in \mathbb{T}}\left|\sigma_{n}^{f}(z)\right| \leq \max _{z \in \mathbb{T}}|f(z)|$ 
holds for every $f \in A(\mathbb{T})$, we obtain

$$
\begin{aligned}
& \max _{z \in \mathbb{T}}\left|\sigma_{2 n}^{p_{n}(z)-z^{n}}(z)\right|=\max _{z \in \mathbb{T}}\left|\sigma_{2 n}^{p_{n}(z)-z^{n}-z^{3 n} q_{n}(z)}(z)\right| \leq \\
& \max _{z \in \mathbb{T}}\left|p_{n}(z)-z^{n}-z^{3 n} q_{n}(z)\right|=\left\|\bar{\chi}^{3} \chi^{1} \xi_{n}-h_{n}\right\|<1 / n,
\end{aligned}
$$

i.e. $\left\|\sigma_{2 n}^{p_{n}(z)-z^{n}}\right\| \rightarrow 0$ as $n \rightarrow \infty$. However, $\sigma_{2 n}^{p_{n}(z)-z^{n}}(z)=\sigma_{2 n}^{p_{n}(z)}(z)-z^{n}(n+1) /(2 n+1)$, and thus $\sigma_{2 n}^{p_{n}(z)-z^{n}}(-1) \rightarrow 1 / 2$ as $n \rightarrow \infty$ for odd $n$, contrary to $\left\|\sigma_{2 n}^{p_{n}(z)-z^{n}}\right\| \rightarrow 0$. Hence $\left\|\bar{\chi}^{3} \chi^{1} \xi_{n}-h_{n}\right\| \nrightarrow 0$ for any $h_{n} \in A_{\Gamma_{+}}$, and therefore $\bar{\chi}^{3} \notin\left(A_{\Gamma_{+}}\right)_{b}^{C(G)}$. The maximality of $A_{\Gamma_{+}}$implies that $\left(A_{\Gamma_{+}}\right)_{b}^{C(G)}=A_{\Gamma_{+}}$, as desired.

Thanks are due to J. Cima, K. Stroethoff and S. Saccone for stimulating discussions.

\section{References}

[1] H. Arizmendi and T. Tonev, Bourgain algebras of topological algebras, Scientific Review 19-20 (1996), 115-118.

[2] J. A. Cima and R. Timoney, The Dunford-Pettis property for certain planar uniform algebras, Michigan Math. J. 34 (1987), 66-104.

[3] B. Cole and T. W. Gamelin, Tight uniform algebras, J. Funct. Anal. 46 (1982), 158-220.

[4] T. W. Gamelin, Uniform Algebras, 2nd ed., Chelsea, New York, 1984.

[5] H. Helson, Analyticity on compact abelian groups, in: Algebras in Analysis (Proc. NATO Advanced Study Inst., Birmingham, 1973), Academic Press, London, 1975, 1-62.

[6] T. Tonev, Big-Planes, Boundaries and Function Algebras, Elsevier and North-Holland, Amsterdam, 1992.

[7] T. Tonev, Bourgain algebras and inductive limit algebras, in: Function Spaces, K. Jarosz (ed.), Contemporary Math. 232, AMS, 1999, 339-344.

[8] T. Tonev and K. Yale, Hankel type operators, Bourgain algebras and isometries, in: Oper. Theory Adv. and Appl. 87, I. Gohberg, P. Lancaster and P. N. Shivakumar (eds.), Birkhäuser, Boston, 1996, 413-418.

[9] K. Yale, Bourgain algebras, in: Function Spaces, K. Jarosz (ed.), Lect. Notes in Pure and Applied Math. 136, Marcel Dekker, New York, 1992, 413-422. 